\title{
Thèses (Leitbild) de la FMH pour le domaine du sauvetage en Suisse
}

Forum du sauvetage*

* Membres du Forum
du sauvetage
Dr Denis Bachmann/SSP,
Dr Sergei Bankoul,
privat-docent/DDPS,
Dr Lion Bernoulli/MU
préhospitalière SSMUS,
Dr Konrad Diem/SSIM,
Dr Bruno E. Durrer/SSMM/
SSMG, Dr Ernst Gähler/
vice-président FMH,
Dr Manuel Rupp/SSPP,
Dr Patrick Siebenpfund/SSMI,
Dr Gianmaria Solari/IAS,
Dr Paul-Martin Sutter/SSC,
Prof. Wolfgang Ummenhofer/
SSAR, Prof. Heinz Zimmer-
mann/ MU hospitalière
SSMUS

Le direttive della FMH per il settore del salvataggio in Svizzera sono disponibili anche in italiano - sulla pagina Web: www.fmh.ch $\rightarrow$ Servizi $\rightarrow$ Piattaforma salvataggio o www.saez.ch $\rightarrow$ Numéro actuel o $\rightarrow 2010 \rightarrow 33$.

Correspondance:

Prof. Wolfgang Ummenhofer Universitätsspital Basel Departement Anästhesie CH-4031 Basel

wummenhofer@uhbs.ch
Le Forum du sauvetage de la FMH est le forum des sociétés de discipline médicale participant à l'assistance préhospitalière d'urgence; c'est pourquoi l'Interassociation de sauvetage et le Service sanitaire coordonné y sont également représentés. Les lignes directrices rédigées d'après le point de vue de la FMH visent à uniformiser les soins professionnels de premiers secours, également en cas de catastrophes et autres événements graves, sur les plans opérationnel et juridique en Suisse.

La FMH s'engage pour des soins urgents associant les médecins d'urgence, ou les médecins de famille spécialement formés, et les ambulanciers.

Les Thèses publiées en 1996 par le Forum du sauvetage de la FMH ont fortement influencé ce domaine en Suisse. Les nouvelles directives doivent répondre aux exigences actuelles pour que le système de secours soit efficace et adapté aux conditions helvétiques.

\section{Médecins de garde comme médecins de premier recours pour soins urgents}

Il relève de la compétence des cantons de garantir sur l'ensemble de leur territoire un service d'urgence, $24 \mathrm{~h}$ sur 24, dont l'exécution est confiée aux sociétés cantonales de médecine. Dans les régions où la géographie ne permet pas de mettre en place un réseau d'urgence sur l'ensemble du territoire, les médecins de premier recours officient comme médecins de garde suite à une formation adéquate dans le cadre d'un concept de sauvetage cantonal. Chaque canton met les fonds à disposition pour l'équipement et les services de garde. Tout médecin de garde doit être capable de reconnaître un patient en danger de mort et de lui prodiguer les soins appropriés jusqu'à l'arrivée du médecin d'urgence. Soumise à un contrôle de qualité, la formation de ces médecins est organisée par le Forum du sauvetage.

Les études de médecine en Suisse doivent aussi transmettre des connaissances et des aptitudes spécifiques aux soins urgents. C'est pourquoi la FMH s'engage pour que le cours de médecin de garde soit intégré au curriculum des facultés de médecine.

\section{Médecins d'urgence}

Les médecins d'urgence doivent être prêts à intervenir à tout moment pour des institutions appropriées, ils doivent être reliés à un réseau de sauvetage reconnu, et il faut aussi faire appel à eux en cas d'indication médicale correspondante, qui peut varier au plan régional selon le lieu et l'heure de l'intervention. Le non-recours aux médecins d'urgence lors d'une indication médicale doit être justifié. Les concepts cantonaux de médecine d'urgence définissent également de manière contraignante les indications d'intervention.

Les médecins d'urgence disposent d'une formation postgraduée spécifique et d'un équipement particulier. L'Institut suisse pour la formation médicale postgraduée et continue (ISFM) règle leur formation postgraduée; la
Société suisse de médecine d'urgence et de sauvetage (SSMUS) décerne l'attestation de formation complémentaire de Médecine d'urgence préhospitalière (médecin d'urgence).

\section{Ambulanciers et techniciens ambulanciers}

La formation d'ambulancier-ère (diplôme EPS) et de technicien-ne ambulancier-ère (diplôme EP) est définie par l'Office fédéral de la formation et de la technologie (OFFT).

Les cas d'urgence graves nécessitent le recours d'ambulanciers diplômés. Leur compétence initiale peut être étendue à une compétence médicale déléguée; l'ambulancier est alors responsable de son exécution. Même de «simples» transports de malades exigent une prise en charge qualifiée. La formation de technicien ambulancier répond aux exigences minimales en matière de qualité pour les transports planifiés de malades sans diagnostic vital.

La formation et le perfectionnement des ambulanciers et techniciens ambulanciers relèvent de la compétence $\mathrm{du}$ «Forum de formation professionnelle du sauvetage» qui assume également la responsabilité professionnelle pour toutes les questions liées à la reconnaissance de la profession.

\section{Centrales d'appels sanitaires d'urgence (CASU)}

Les CASU coordonnent les interventions préhospitalières de premiers secours sur le plan interrégional. Regroupées en réseau, elles gèrent gratuitement le numéro d'urgence sanitaire 144 sur l'ensemble du territoire. Les ambulanciers, soumis au secret professionnel, disposent d'une formation spécifique aux CASU dont la direction médicale incombe à un médecin d'urgence SSMUS.

Les CASU trient tous les appels d'urgence et organisent de façon géographiquement appropriée les premiers secours.

Selon la situation

- la personne qui demande de l'aide recevra des instructions concrètes afin d'attendre dans les meilleures conditions l'arrivée des équipes de secours,

- le patient en détresse sera renvoyé au médecin de garde ou à un cabinet d'urgence,

- l'intervention de la prochaine ambulance et, en cas d'indication médicale, du médecin d'urgence, ou éventuellement du médecin de garde sera déclenchée,

- l'intervention de l'hélicoptère de secours sera déclenchée en cas d'indication médicale,

- l'intervention directe d'un médecin d'urgence (sauvetage aérien) sera déclenchée, en particulier dans les cas d'urgence pédiatrique graves,

- le recours à l'ambulancier chef des secours (ACS) et au médecin-chef des secours (MCS) est déclenché,

- la CASU sert de dispositif de mobilisation des services sanitaires (backoffice) en cas d'événement grave et de catastrophe. 


\section{Services d'urgence des hôpitaux -}

\section{Médecine d'urgence hospitalière}

Tous les hôpitaux suisses dotés de services d'urgence sont classés en catégories selon des critères uniformes. Cette classification comprend les capacités des établissements et les disciplines, y compris la pédiatrie/chirurgie pédiatrique, permettant la prise en charge de patients nécessitant des soins d'urgence.

La direction médicale et l'organisation d'un service d'urgence doivent être assumées par un médecin titulaire de l'attestation de formation complémentaire (AFC) en médecine d'urgence hospitalière. La FMH a reconnu cette AFC et délégué la formation postgraduée et continue à la SSMUS.

\section{Amélioration de la chaîne de sauvetage}

Les patients nécessitant des soins d'urgence sont pris en charge de manière coordonnée par des profanes, des secouristes ou «first responder» et des équipes professionnelles de sauvetage; c'est ce qu'on appelle la «chaîne de sauvetage».

Des améliorations s'imposent particulièrement en matière de formation des profanes: tout citoyen devrait être formé aux premiers secours, connaître les mesures immédiates pour sauver la vie ainsi que les techniques de réanimation. Cette formation devrait être intégrée à l'enseignement scolaire. Relevant de la compétence du Swiss Resuscitation Council (SRC), la formation des citoyens doit être organisée de manière uniforme et progressive.

\section{Conduite sanitaire en cas d'événement majeur}

Pour la gestion des accidents majeurs et des catastrophes, le Service sanitaire coordonné (SSC) et la SSMUS ont mis en place la plateforme nationale de formation postgraduée "Conduite sanitaire en cas d'événement majeur» (CEFOCA-SFG). Les ambulanciers doivent suivre une formation de chef des secours (CS) ou d'ambulancier chef des secours (ACS) et les médecins d'urgence une formation de médecin-chef des secours (MCS), chacune suivie d'une formation continue obligatoire.

Il incombe aux cantons de mettre en place ces fonctions et de les doter des ressources humaines et matérielles nécessaires.

En cas d'accident, le système d'information informatique (SII) du SSC fournit des informations sur les ressources hospitalières disponibles et soutient le transfert de données entre le lieu de l'accident et les unités de soins. Pour rendre le SII opérationnel, les services et hôpitaux impliqués doivent recevoir les instructions nécessaires et bénéficier régulièrement d'exercices de simulation.

\section{Normes et contrôles de qualité / Protection des données}

\subsection{Délai d'intervention pour les structures} professionnelles de sauvetage

Les patients en danger de mort doivent recevoir en Suisse des secours professionnels dans un délai de 15 minutes (délai d'intervention); dans la mesure du possible, ce délai devra être réduit à 10 minutes. En cas d'indication médicale, un médecin d'urgence doit pouvoir arriver auprès du patient dans le même laps de temps. En régions périphériques, un dispositif de garde peut venir compléter le réseau des médecins d'urgence. Le système de sauvetage local doit être relié aux structures de sauvetage interrégionales (sauvetage aérien).

\subsection{Directeur médical du service de sauvetage}

Chaque service de sauvetage dispose d'un directeur médical titulaire de l'attestation de formation complémentaire en médecine d'urgence de la SSMUS. Ce dernier est habilité à donner des instructions dans le domaine médical, peut déléguer des compétences médicales aux ambulanciers et prend les mesures visant à garantir la qualité du service de sauvetage.

\subsection{Hôpital cible}

Les patients en détresse doivent être autant que possible directement transportés, sous surveillance et traitement d'un médecin qualifié, dans un hôpital adéquat pour la prise en charge définitive des lésions les plus graves. Chaque transport sera effectué avec les ressources en personnel (ambulancier, technicien ambulancier, médecin d'urgence) et techniques (ambulance, hélicoptère) appropriées. Le directeur médical d'un service de sauvetage établit un concept d'intervention pour les hôpitaux de son secteur géographique. Des moyens de sauvetage aérien seront à privilégier en cas de longs trajets par la route.

\subsection{Contrôle de qualité}

Tous les niveaux du sauvetage doivent satisfaire à un contrôle de qualité.

L'IAS édicte des directives pour les moyens de transport et les équipements de services de sauvetage. Tous les services de sauvetage en Suisse doivent être certifiés IAS ou attester d'un contrôle de qualité équivalent. Les coûts supplémentaires imputés aux services de sauvetage certifiés doivent apparaître dans la structure tarifaire des assureurs.

Sur mandat de l'ISFM, la SSMUS est chargée de la formation postgraduée en médecine d'urgence. Le contrôle de qualité des cours de base en médecine d'urgence incombe, en revanche, au Forum du sauvetage (médecin de garde) ou à la SSMUS (médecins d'urgence, MCS).

\subsection{Dispositions relatives à la protection} des données

Une gestion judicieuse de la qualité exige le contrôle des interventions de sauvetage sur la base de données pertinentes qui, actuellement en Suisse, ne sont pas accessibles compte tenu de la législation en matière de protection des données. En collaboration avec les sociétés de discipline médicale, des institutions, des hôpitaux et des organisations de sauvetage, le Préposé fédéral à la protection des données est invité à développer des possibilités de saisie des données caractéristiques sous forme anonyme. 